\title{
Vom Indent zum Scratch - Nanomechanische Tests
}

\author{
Marcel Mück, Alexander Soshnikov, Bodo Wolf, Asta Richter
}

\section{Einleitung}

Da Bauteile immer kleiner und Beschichtungen immer dünner werden, erlangt die direkte und genaue Bestimmung mechanischer Eigenschaften im Nanobereich immer größere Bedeutung. Versteht man die Effekte im Nanobereich, wird es einfacher, auch Effekte im Makrobereich vorherzusagen. Damit bekommen die entsprechenden Messmethoden eine entscheidende Bedeutung bei der Entwicklung der Materialien und Herstellungsmethoden im Hinblick auf optimierte Produkteigenschaften [1]. Aus diesem Grund entwickelte sich jenseits der klassischen mechanischen Prüfverfahren, welche in der Makrodimension angesiedelt sind, in den letzten fünf Jahren ein neuer Zweig der Werkstoffprüfung, der sich mit den Materialeigenschaften im Nanobereich beschäftigt. Dies ist von grundlegender Bedeutung für die Nanotechnologie.

Unter Nanotechnologie fasst man die Manipulation einzelner Atome und Moleküle zusammen mit dem Ziel, im weitesten Sinne neue Werkstoffe mit Strukturen im Nanometer-Bereich zu erzeugen: Hochdichte Datenspeicher für superschnelle Computer, funktionelle superharte Beschichtungen, extrem feste molekulare Klebeverbindungen, effektive Katalysatoren und Membranen oder hoch selektiv wirkende Pharmazeutika sind einige Beispiele dafür [2].

In diesem Bericht sollen zwei Geräte in Bezug zueinander gesetzt werden, die die Messung verschiedener nanomechanischer Eigenschaften erlauben. Dabei handelt es sich um das TriboScope (Hysitron Inc.) [3] als Aufsatz für ein Rasterkraftmikroskop (SFM) und das Nanoscan (Technological Institute for Superhard and Novel Carbon Materials; Troitsk/Russland) [4], dargestellt in den Abbildungen 1 und 2. Diese ermöglichen die Messung von Härte und E-Modul einer Oberfläche.

Zum Vergleich beider Geräte soll das Augenmerk auf die Bestimmung der Nanohärte gerichtet werden. Bei deren Ermittlung werden zwei unterschiedliche Wege beschritten. Das TriboScope verwendet die klassische Methode eines Indents, während das Nanoscan mittels eines Scratches (=Ritzes) die Bestimmung der Härte ermöglicht. Diese unterschiedlichen Herangehensweisen und die daraus resultierenden Ergebnisse sollen in den folgenden Abschnitten dokumentiert werden.

\section{Aufbau und Funktion des TriboScopes und des Nanoscans}

\subsection{Allgemeine Betrachtungen}

Beide hier beschriebenen Messverfahren bieten die Möglichkeit, mittels einer Sonde (= Spitze) die Oberfläche abzutasten und damit ein Höhenprofil zu messen. Gleichzeitig kann dieselbe Tastspitze zur Bestimmung der mechanischen Eigenschaften genutzt werden. Veränderungen der Oberfläche liegen beim TriboScope als Indent und beim Nanoscan als Scratch vor.

\subsection{TriboScope}

Das TriboScope [3] ist ein Zusatzmodul (Abb. 1a) für ein SFM (Abb. 1b). Es ersetzt dessen Standardmikroskopkopf

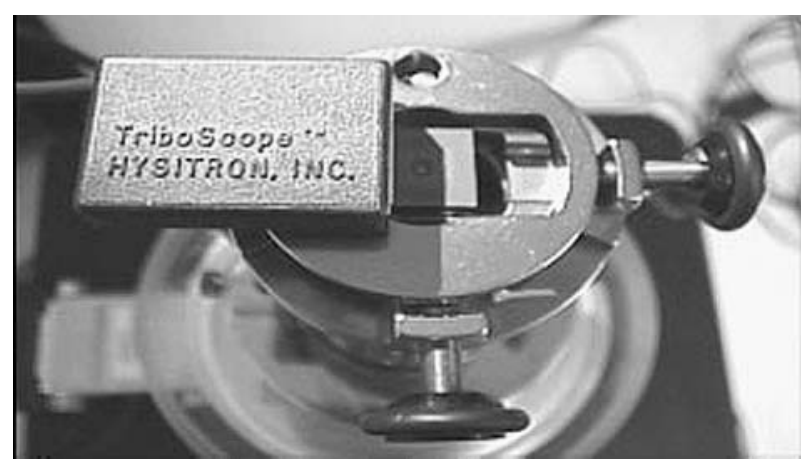

Abb. 1a: TriboScope der Firma Hysitron Inc. als Aufsatz für das SFM

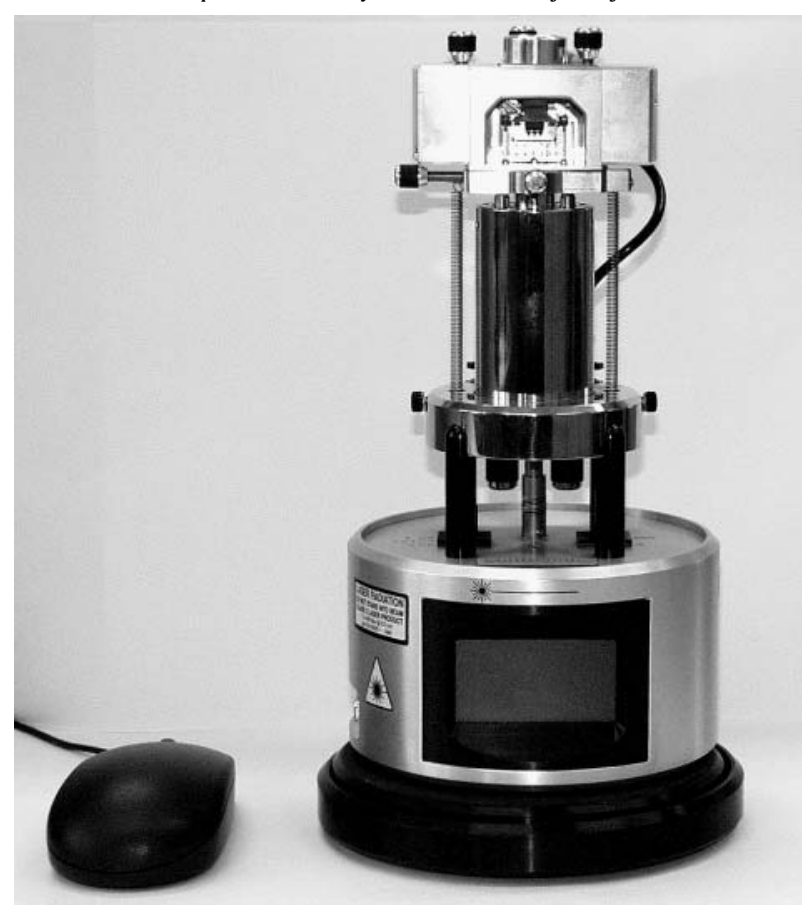

Abb. 1b: Ansicht des Multimode SFM der Firma Veeco Instruments Inc. 


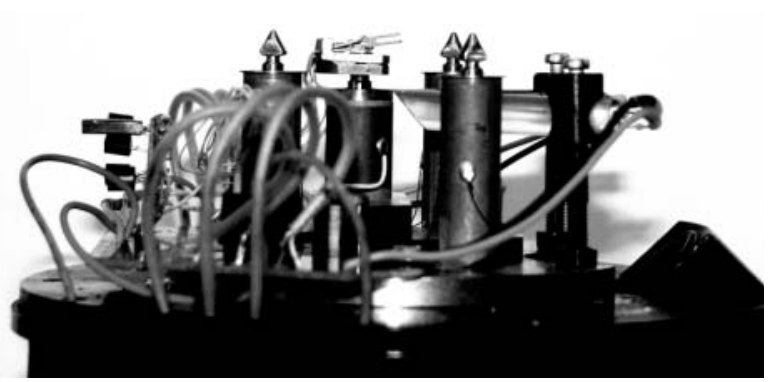

Abb. 2a: Geöffnetes Gehäuse des Nanoscans; zu erkennen sind die drei Auflagepunkte für die Probe und der doppelarmige Cantilever.

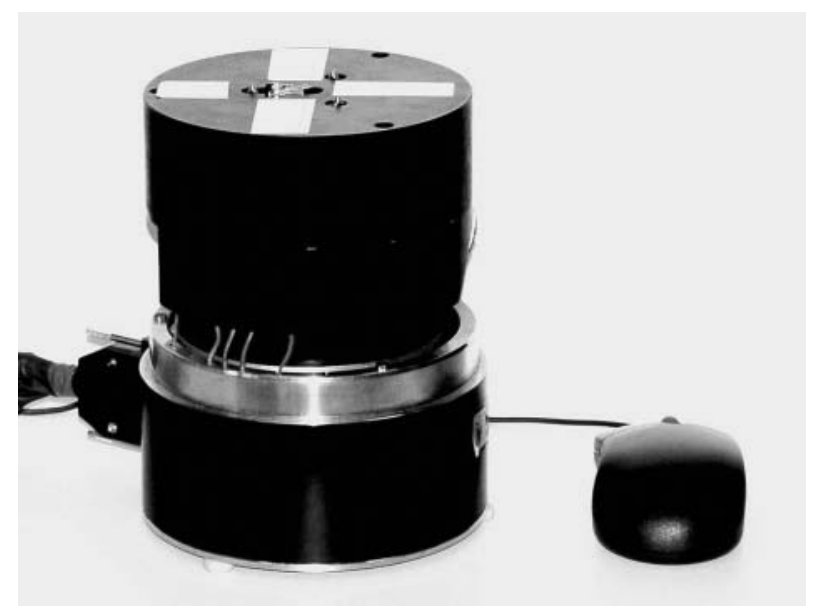

Abb. 2b: Ansicht des Geräts Nanoscan vom Technological Institute for Superhard and Novel Carbon Materials in Troitsk, Russland

und nutzt die vorhandene Hard- und Software zur Umsetzung der Messwerte in ein Oberflächenprofil. Das Prinzip der Abtastung unterscheidet sich dabei aber grundsätzlich von dem eines Rasterkraftmikroskops. Bei diesem wird das Oberflächenprofil durch eine Nadel an einem biegsamen Cantilever (= Federbalken) abgetastet. Durch ein optisches System wird die Auslenkung der Nadel registriert und der somit detektierte Höhenunterschied der Oberfläche als elektrisches Signal erfasst. Über eine elektronische Rückkopplungsschleife wird dieses Signal auch an den Piezokristallträger der Probe uibermittelt, der dann eine vertikale Probensteuerung vornimmt. Dadurch wird die Probe in einem konstanten Abstand unter der Nadel entlanggefuihrt. Die Z-Steuerung des Piezokristalls kann auch als Abtastsignal abgebildet werden und enthält die wahren Höhenwerte des Probenprofils [5-8].

Das Kernstiick des TriboScopes ist ein 3-Plattenkondensator. An dessen mittlerer, beweglicher Platte, welche mit je zwei Federn an den äußeren Platten befestigt ist, befindet sich eine Diamantspitze (Abb. 3). Zwischen der mittleren und den äußeren Platten wird jeweils eine Wechselspannung angelegt. Beide Spannungen $\left(\mathrm{U}_{1}, \mathrm{U}_{2}\right)$ haben den gleichen Betrag, sind aber um $180^{\circ}$ phasenverschoben.

Um eine Oberfläche abzubilden, wird diese an die Nadel herangefahren, bis eine Auslenkung der beweglichen Kondensatorplatte erfolgt. Als Folge dessen ändert sich das Spannungsverhältnis zwischen $\mathrm{U}_{1}$ und $\mathrm{U}_{2}$. Der Piezoaktuator, auf dem sich die Probe befindet, wird nun so justiert, dass die Differenz beider Spannungen nahe Null

\section{3-Plattenkondensator}

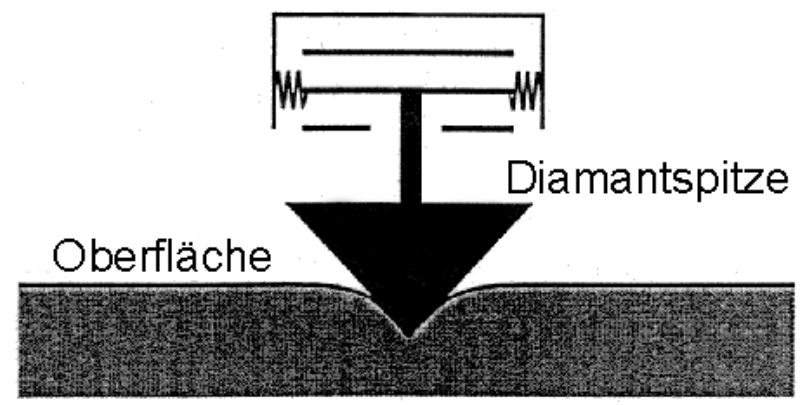

Abb. 3: Aufbau und Funktionsweise des TriboScopes

wird. Dies ergibt dann den Nullpunkt als Bezug für die Höhenmessungen.

Die Abbildung des Oberflächenprofils erfolgt nun zeilenweise, ähnlich wie beim Bildaufbau eines Monitors oder TV-Geräts. Dabei verschiebt der Piezoaktuator die Probe in $\mathrm{x}$ - und $\mathrm{y}$-Richtung unter der Nadel. Die sich ändernden Spannungsverhältnisse der Kondensatorplatten werden als Signal in ein Strombild umgewandelt und uibernehmen die Steuerung der Piezoaktuatoren. Diese verfahren die Probe so, dass die bewegliche Kondensatorplatte wieder in ihre Nulllage gebracht wird. Das Höhenbild entsteht durch das gleiche Prinzip wie beim oben beschriebenen SFM.

Zur Messung der Härte wird ein Indent erzeugt. Dazu wird die Diamantspitze an der gewünschten Stelle positioniert. Durch eine gezielte Änderung der Spannung lässt sich nun über eine Kondensatorplattenbewegung eine definierte Kraft auf die Oberfläche ausüben. Diese wird bestimmt durch:

$F=0.5 \varepsilon A\left(\frac{U}{d}\right)^{2} \sim U^{2}$.

$U$ entspricht dabei der Spannung zwischen einer der äußeren Kondensatorplatten und der beweglichen Mittelplatte, $A$ entspricht dem Plattendurchmesser, $\varepsilon$ der Dielektrizitätskonstante und $d$ dem Abstand der Platten.

Mittels der Steuerungssoftware werden die maximale Kraft sowie der zeitliche Verlauf des Krafteintrags vorgegeben. Während des Indentens wird ein Kraft-Weg-Diagramm aufgenommen (Abb. 4). Dieses stellt die Belastung und Entlastung der Probe als eine Funktion der Eindringtiefe dar. Die Steuerungssoftware gibt als Ergebnis aus diesem Diagramm die Härte nach folgenden Formeln aus [9]:

$F=K\left(h-h_{f}\right)^{m}$

$h_{c}=h_{\max }-0.75 \frac{F_{\max }}{S}$

$H=\frac{F_{\max }}{A\left(h_{c}\right)}$

Um die Kraft $F$ in Abhängigkeit von $h$ zu bestimmen, wird die Gleichung 2 verwendet. Der Parameter $h$ ist hier die 


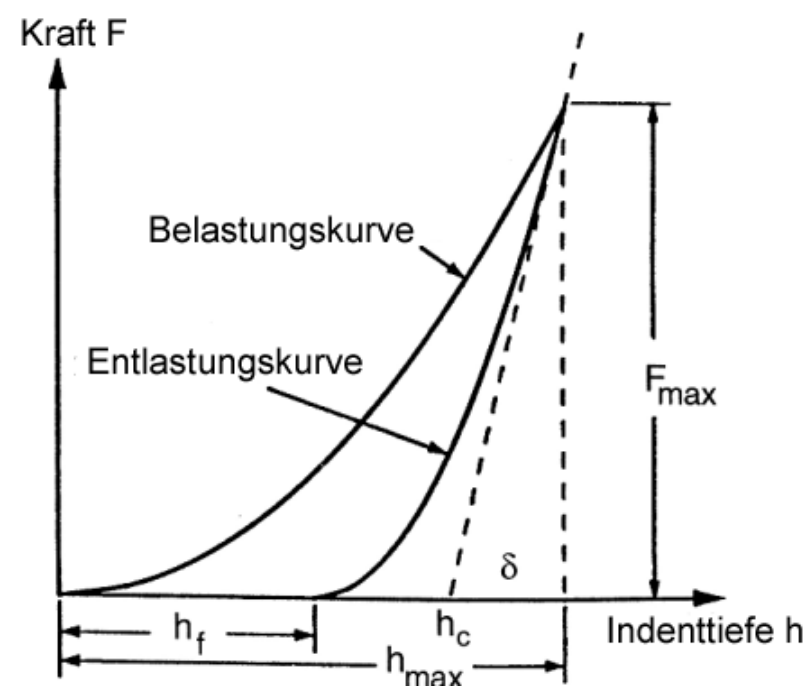

Abb. 4: Schematisiertes Kraft-Weg-Diagramm [9]

Eindringtiefe, $h_{f}$ die Tiefe des verbleibenden Eindrucks, $K$ ist eine Fitkonstante und $m$ der Fitexponent, welcher zwischen 1 und 2 liegt. Um nun die Härte zu ermitteln, wird Gleichung 4 benötigt. In dieser wird die maximale Kraft $F_{\max }$ durch die Funktion der Kontaktfläche $A\left(h_{c}\right)$ geteilt. Aus Gleichung 3 kann die Kontakttiefe $h_{c}$ bestimmt werden. Als Parameter in diese Berechnung fließen die maximale Eindringtiefe $h_{\max }$, die maximale Kraft $F_{\max }$ an der Stelle $h_{\max }$ und die Steifheit $S=(d F / d h)_{h=\max }$ ein. Als Ergebnis aus Gleichung 4 erhält man die Härte in GPa.

\subsection{Nanoscan}

Das Nanoscan [4] ist ein SFM, welches im dynamischen Kontaktmodus mittels oszillierender Spitze arbeitet, ähnlich dem Tapping-Modus [5-8]. Das Nanoscan wird zum Scratchen von Materialien unterschiedlichster Festigkeit benutzt. Es können auch Materialeigenschaften wie Haftfestigkeit, Materialermuidung und Reibungsverhalten ermittelt werden. Kernstuick dieses Gerätes ist ein doppelarmiger piezokeramischer Cantilever, an dem eine Spitze aus Diamant bzw. Fullerit befestigt ist (Abb. 2a). Die Spitze kann mittels eines Piezodrives von unten an eine Probe herangefahren werden. Sie wird auf drei Punkten gelagert, die mittels mehrerer Piezokristalle in $\mathrm{x}$ - und $\mathrm{y}$ Richtung bewegt werden können. Das ganze System ist in einem zylindrischen Gehäuse (Abb. 2b) untergebracht und auf einer schwingungsisolierten Plattform befestigt.

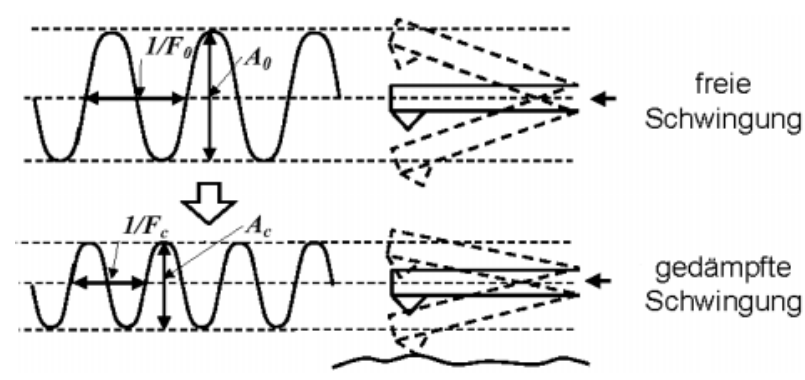

Abb. 5: Schematischer Verlauf der freien und gedämpften Schwingung bei Annäherung der Sonde an die Oberfläche; $A_{0}$ und $F_{0}$ entsprechen der Amplitude und Frequenz der freien, $A_{C}$ und $F_{C}$ der Amplitude und Frequenz der gedämpften Schwingung.
Um eine Oberfläche abzubilden, wird diese genau wie beim TriboScope nach dem Rasterprinzip abgetastet. Der piezokeramische Cantilever wird zum Oszillieren gebracht. Trifft die Spitze die Oberfläche, verringert sich die Amplitude und mit zunehmendem Maße auch die Frequenz. Es handelt sich dabei, wie in Abbildung 5 dargestellt, um eine gedämpfte Schwingung.

Eine Rückkopplungsschaltung misst nun entweder die Änderung der Amplitude oder die der Frequenz und regelt diese auf den ursprünglichen Wert zurück. Diese Änderung wird aufgenommen und ist ein Maß für die Höhe der Oberfläche an der gemessenen Stelle. Der für dieses Signal genutzte Parameter entscheidet darüber, was für Informationen sich aus dem Höhenbild ablesen lassen (Abb. 6). Wird die Amplitude als Steuersignal verwendet, so erhält man das Profil der viskosen, bei Nutzung der Frequenz das der elastischen Oberfläche [4].

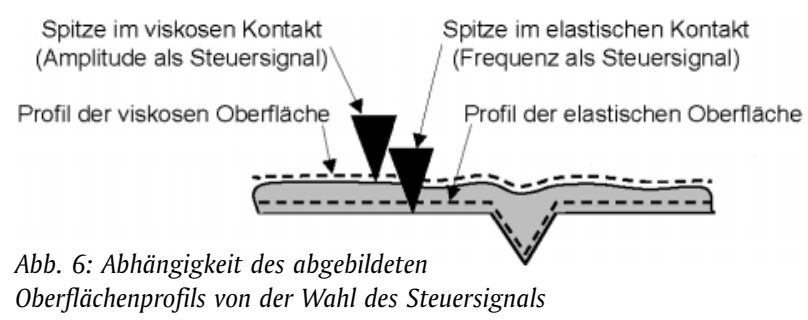

Um die Härte einer Oberfläche zu messen, wird in ihr ein Scratch erzeugt. Mit einer bestimmten Kraft dringt die Spitze in das Material ein und wird anschließend in eine Richtung mit dem Scanner über eine festgelegte Strecke bewegt. Aus der Breite $b$ des Scratches wird die Härte $H$ nach folgender Formel berechnet:

$H=\frac{k F}{b^{2}}$

Der Parameter $k$ ist dabei ein Formfaktor fuir die Spitze. Dieser kann mittels eines Materials ermittelt werden, von dem die Härte bekannt ist. Dazu wird im Eichmaterial ein Scratch mit der Kraft $F_{1}$ und der Breite $b_{1}$ erzeugt. Nun lässt sich $k$ ausrechnen.

Bedingung für den Vergleich zweier Messungen ist, dass die Eindringtiefe der Spitze und damit die Breite des Scratches gleich sind, da sich ansonsten $k$, aufgrund der veränderten Spitzengeometrie, ändern würde. Also kann dieser Koeffizient, bei gleichbleibender Breite $b$ des Scratches, als konstant angenommen werden, womit sich folgende Formel ergibt:

$H=\frac{H_{1} b_{1}^{2} F}{F_{1} b^{2}}$.

Zur Härtemessung mit dem Nanoscan muss also ein Referenzmaterial herangezogen werden, da es sich bei der Formel zur Härtebestimmung um eine Gleichung mit zwei unbekannten Variablen handelt.

Bei der Bestimmung der Härte mit dem Nanoscan handelt es sich um ein vergleichendes Verfahren, welches keine absolute Härtemessung erlaubt. 


\subsection{Zusammenfassung}

Beide Geräte bieten die Möglichkeit, eine Oberfläche topographisch darzustellen und an einer gewählten Stelle die Härte zu messen. Das TriboScope nutzt für die Abtastung und den Indent ein 3-Platten-Kondensatorsystem, das Nanoscan eine piezoelektronische Sonde. Ersteres kann eine absolute Härtemessung durchführen, während letzteres ein Referenzmaterial benötigt. Der Vorteil des Nanoscans liegt darin, dass Spitzen mit beliebiger Geometrie verwendet werden können, während die für das TriboScope eine festgelegte Form aufweisen müssen, da diese in die Berechnung einfließt. Für das TriboScope wird zum Beispiel eine dreiseitige Spitze mit $90^{\circ}$ Öffnungswinkel verwendet [10].

\section{Härtmessung an Lime Glas, Quarz und Saphir}

\subsection{Vorbetrachtungen}

Im Folgenden soll auf die Bestimmung der Härte von Lime Glas, fused Quarz (gleichzeitig Referenzprobe für Nanoscan) und Saphir mittels des TriboScopes und des Nanoscan eingegangen werden.

\subsection{Messung der Härte mit dem Nanoscan}

Wie unter 2.2 beschrieben, ist eine Standardprobe erforderlich, um die Härte eines unbekannten Materials zu messen. Dafuir wurde eine isotrope Milchquarzprobe (= fused Quarz) mit einer angegebenen Härte von $10 \mathrm{GPa}$ gewählt. In diesem Material wurden zwei Scratche (Abb. 7a) mit einer Kraft von 0,5 mN und 0,6 mN erzeugt und deren Breite $b$ gemessen. Dazu wurde mit der zugehörigen Auswertungssoftware ein Profilschnitt (Abb. 7b) durch das zweidimensionale Abbild der Scratche gelegt. Für den Profilschnitt errechnet die Software dann aus den Grauwerten ein Höhenprofil, wo auch die Ritzbreite abgelesen werden kann.
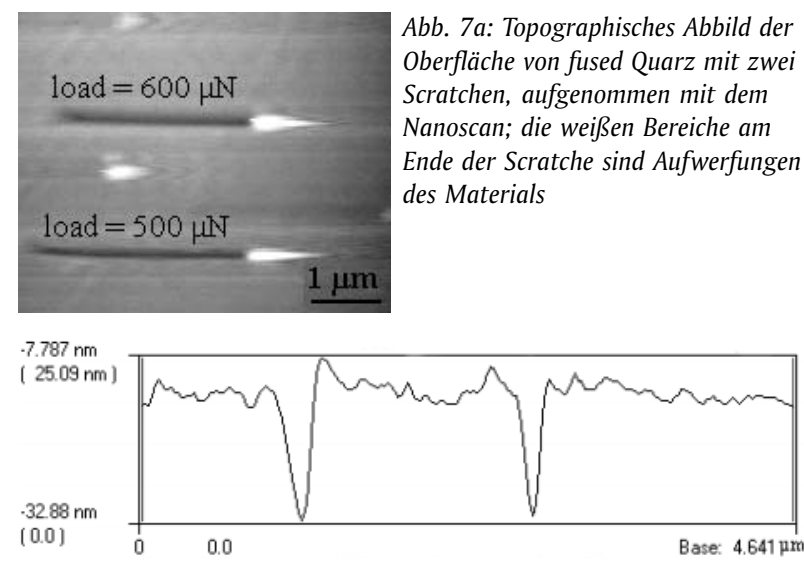

Abb. 7b: Profilschnitt senkrecht zu den Scratchen

In den folgenden Scratchtests wurden in Saphir und Lime Glas Scratche erzeugt, die möglichst die gleiche Breite $b$ erreichen. Versuche mit unterschiedlichen Kräften waren erforderlich. Der Scratch mit der am nächsten an den Referenzwert heranreichenden Breite wurde dann ausgewählt und die Parameter $F$ und $b$ in die Berechnung nach Gleichung (5) übernommen. Saphir wurde aufgrund seiner hohen Festigkeit mit $1 \mathrm{mN}$ anstatt $0,5 \mathrm{mN}$ geritzt, um die Oberfläche gut sichtbar zu modifizieren.

\subsection{Messung der Härte mit dem TriboScope}

Da mit dem TriboScope eine absolute Härtemessung möglich ist, wurden alle drei Proben vermessen. Es wurden die Kraft-Weg-Kurven für mehrere aufeinanderfolgende Indents, sogenannte Multiindents [11], aufgenommen. Dabei wurde vom gleichen Messort eine vorher festgelegte Anzahl von Indents mit steigender Kraft bis maximal $6 \mathrm{mN}$ durchgeführt, um schnell und effektiv eine größere Menge von Messwerten zu erhalten. Aus ihnen ermittelte die Software dann die Härtewerte in GPa. Ein Beispiel für einen Indenteindruck, aufgenommen mit dem TriboScope, ist in Abbildung 8 zu sehen.

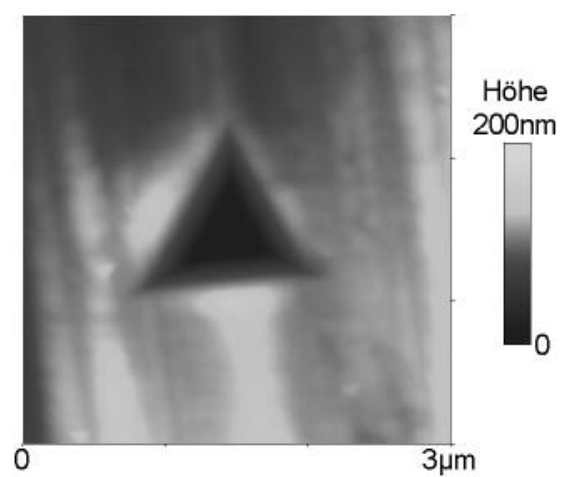

Abb. 8: Abbildung eines typischen Indenteindrucks mit einer Kraft von $4 \mathrm{mN}$

\subsection{Ergebnisse und Auswertung}

In der Tabelle 1 sind die Ergebnisse der Härtemessung für das Nanoscan zusammengestellt. Bei der NanoscanMessung wurde in fused Quarz eine durchschnittliche Scratchbreite von $190 \mathrm{~nm}$ erzielt. In Saphir konnte diese mit einer geringen Abweichung des Mittelwertes von $b$ bezogen auf den Wert von fused Quarz (193 nm zu $190 \mathrm{~nm}$ ) relativ genau erreicht werden. In Lime Glas ist die Abweichung mit $216 \mathrm{~nm}$ größer.

\begin{tabular}{|r|r|r|r|}
\hline Messung & $\begin{array}{c}\text { fused Quarz b [nm] } \\
\mathbf{F = 0 , 5} \mathbf{~ m N ~}\end{array}$ & $\begin{array}{c}\text { Lime Glas b [nm] } \\
\mathbf{F = 0 , 5} \mathbf{~ m N ~}\end{array}$ & $\begin{array}{c}\text { Saphir b [nm] } \\
\mathbf{F}=1 \mathbf{~ m N}\end{array}$ \\
\hline 1 & 204 & 221 & 204 \\
2 & 187 & 198 & 198 \\
3 & 202 & 205 & 195 \\
4 & 184 & 236 & 210 \\
5 & 175 & 207 & 191 \\
6 & 178 & 218 & 178 \\
7 & 201 & 229 & 181 \\
8 & 184 & 214 & 184 \\
9 & 197 & 207 & 202 \\
10 & 187 & 225 & 187 \\
\hline Mittelwert: & $(190 \pm 15)$ & $216 \pm 20$ & $193 \pm 17$ \\
\hline Härte [GPa]: & -7.7 & 19,4 \\
\hline
\end{tabular}

Tab. 1: Ergebnisse für die Scratchbreite $b$ und die Härte H der NanoscanMessungen

Nach Gleichung 6 wurde die Härte berechnet. Für Saphir ergab sich ein Härtewert von 19,4 GPa, der deutlich unter dem mit dem TriboScope gemessenen Wert von 36,8 GPa liegt. Die für Lime Glas ermittelte Härte von 7,7 GPa hingegen liegt im erwarteten Bereich. Die hohe Diskrepanz des Wertes für Saphir kann unter Umständen darauf zurïckgefuihrt werden, dass das Referenzmaterial 


\begin{tabular}{|c|c|c|c|c|c|}
\hline $\mathbf{h}$ [nm] & H [Gpa] & $h$ [nm] & H [Gpa] & $\mathrm{h}$ [nm] & $\mathrm{H}$ [Gpa] \\
\hline 46 & 7,9 & 33 & 10,4 & 22 & 34,0 \\
\hline 87 & 7,6 & 44 & 10,6 & 30 & 38,3 \\
\hline 122 & 7,4 & 56 & 10,6 & 40 & 38 \\
\hline 155 & 7,1 & 65 & 10,5 & 49 & 38 \\
\hline 183 & 7,0 & 77 & 10,1 & 56 & 39, \\
\hline 208 & 7,0 & 89 & 10,2 & 66 & 38,4 \\
\hline 233 & 6,9 & 98 & 9,7 & 76 & 37,2 \\
\hline 254 & 6,9 & 107 & 9,6 & 86 & 35,6 \\
\hline 275 & 6,8 & 117 & 9,4 & 93 & 35,7 \\
\hline 296 & 6,7 & 138 & 9,3 & 109 & 35,3 \\
\hline 339 & 6,5 & 157 & 9,3 & 137 & 34,5 \\
\hline Mittelwert: & 7,1 & Mittelwert: & 9,9 & Mittelwert: & 36,8 \\
\hline $\begin{array}{l}\text { Abweichung } \\
\text { vom Mittelwert: }\end{array}$ & $\pm 0,8$ & $\begin{array}{l}\text { Abweichung } \\
\text { vom Mittelwert: }\end{array}$ & $\pm 0,7$ & $\begin{array}{l}\text { Abweichung } \\
\text { vom Mittelwert: }\end{array}$ & $\pm 2,9$ \\
\hline
\end{tabular}

Tab. 2: Härtewerte aufgenommen mit dem TriboScope in Abhängigkeit von der Indenttiefe für (a) Lime Glas (lks.), (b) fused Quarz (Mi.), (c) Saphir (re.)

von der Härte her zu weit vom getesteten Material entfernt liegt. Das Prinzip der vergleichenden Härtebestimmung eignet sich besonders fuir Proben geringer Härtedifferenz.

In Tabelle 2 sind die Ergebnisse für die Multiindents in Saphir, Lime Glas und fused Quarz mit dem TriboScope zusammengestellt. Auffällig ist die Tendenz, dass die Härte $H$ mit zunehmender Tiefe $h$ im Messbereich abnimmt. Dies resultiert daraus, dass die Härte keine Materialeigenschaft an sich ist, sondern durch die Realstruktur bestimmt wird. In kleinen Deformationsvolumina, wie für die Nanoindentation charakteristisch, ist die Anzahl vorhandener Gitterbaufehler gering und wird beim Indentieren erhöht. Mit zunehmender Dichte von Versetzungen wird zunächst die Möglichkeit des Materialtransports vereinfacht, bis bei hohen Versetzungsdichten eine gegenseitige Blockierung eintritt. Dies verdeutlicht sich in dem Verlauf der tiefenabhängigen Härte.

Der gerundete Mittelwert der Härte von fused Quarz entspricht genau den bekannten 10 GPa. Die Ergebnisse fuir Saphir und Lime Glas sind ebenfalls positiv zu beurteilen und entsprechen jeweils den Erfahrungswerten. Die Streuung der Messwerte bewegt sich im normalem Rahmen von fünf bis zehn Prozent.

\subsection{Zusammenfassung}

In den Versuchen konnte gezeigt werden, dass die Vergleichbarkeit der ermittelten Härtewerte beider Geräte nicht ohne weiteres gegeben ist. Eine Möglichkeit für die unterschiedlichen Messergebnisse liegt in den verschiedenen Messmethoden. Die Methode der Indentation wird dem Scratchtest gegenuibergestellt. Für einen direkten Vergleich müssen die theoretischen Beschreibungsmodelle speziell fuir das Nanoscan weiterentwickelt werden.

Die mittels Nanoscan ermittelte Härte von Saphir unterscheidet sich von der vom TriboScope ermittelten um ca. 17 GPa. Gute Übereinstimmung ergab sich bei Lime Glas, wo die Differenz beider Messungen bei nur rund 0,7 GPa lag.

Da es sich beim TriboScope um ein kalibriertes Gerät handelt und eine Vielzahl bestätigter Ergebnisse vorliegen [3, 9-11], sollte für zukünftige Arbeiten der Fokus auf die Optimierung und Kalibrierung des Nanoscan gerichtet werden.

\section{Ausblick}

Eine zukünftige Anwendung beider hier vorgestellter Geräte soll die qualitative und quantitative Untersuchung von neuartigen Kompositwerkstoffen sein. In dem ProInno-Projekt, in dessen Rahmen das Nanoscan entwickelt wurde, geht es dabei speziell um die Charakterisierung von Diamantschleifpads, bei denen Diamanten in eine Metall-Matrix eingebettet werden. Es handelt sich dabei um ein Kooperationsprojekt zwischen der Technischen Fachhochschule Wildau und der Vollstädt Diamant GmbH mit dem Ziel, neuartige Schleifkörper, zum Beispiel fuir Optiken, zu entwickeln und im Markt zu platzieren.

Mit Hilfe von Scratchtests soll die Qualität der Einbindung der Diamantkörper in das Trägermaterial getestet werden, bevor Feldversuche durchgefuihrt werden. Ebenfalls zu ermitteln ist die Festigkeit der Schleifpads. TriboScope und Nanoscan eignen sich fuir diese Untersuchungen.

Die Verwendung einer ultraharten Fulleritspitze beim Nanoscan ermöglicht insbesondere das Scratchen von sehr harten Materialien (->Diamant). Somit eröffnen sich fuir die Zukunft noch weitere Anwendungsgebiete für dieses Gerät auch außerhalb des Projektes.

\section{Danksagungen}

Die Forschungsarbeit wurde durch die „Arbeitsgemeinschaft industrieller Forschungsvereinigungen“ (AiF) im Programm „Innovationskompetenz mittelständischer Unternehmen“ (ProInno KF 0132808 KUK2) gefördert und finanziert. Marcel Mück und Bodo Wolf danken ProInno für die finanzielle Unterstuitzung.

Alexander Soshnikow fuihrte Untersuchungen im Rahmen des Programms „Internationale Qualitätsnetzwerke - IQN“ durch, welches durch den deutschen Akademischen Austauschdienst (DAAD) und das Zukunftsinvestitionsprogramm Deutschlands (zip) gefördert wird. Er dankt dem DAAD und zip für die Förderung des Auslandsaufenthalts an der TFH Wildau, wo diese Untersuchungen durchgefuihrt wurden.

Für tatkräftige Hilfe und wissenschaftliche Diskussionen danken wir Herrn Dipl.-Krist. R. Ries, TFH Wildau und Herrn Dr. K. Gogolinski, Technological Institute for Superhard and Novel Carbon Materials, Troitsk.

\section{Literaturverzeichnis}

[1] Spectrum LOT 85 (2001) S. 6.

[2] Physikalische Blätter 55 (1999) S. 57-61.

[3] Hysitron Inc., TriboScope User Manual 1997.

[4] Nanoscan Measurement User Manual 2001.

[5] Ernst Meyer, Atomic Force Microscopy: Fundamentals to Most Advanced Applications, Springer New York 2002, ISBN 3540431802.

[6] A. De Stefanis/A. A. G. Tomlinson, Scanning Probe Microscopies: from Surface Structures to Nano-Scale Enginee- 
ring, Trans Tech Publications Ltd. Zürich 2001, ISBN 0878498680.

[7] http://www.veeco.com/html/library.asp.

[8] A. Richter and R. Smith, Scanning Probe Microscopy and Computer Simulations, Crystal Research \& Technology, 38 (3-5) (2003).

[9] W. C. Oliver and G. M. Pharr, J. Mat. Res. 7 (1992) 1564.

[10] A. Richter, R. Ries, R. Smith, M. Henkel and B. Wolf, „Diamond and Related Materials" 9 (2000) 170-184.

[11] B. Wolf and A. Richter, New Journal of Physics 5 (2003) 15.1-15.17.

[12] http://www.mtu-net.ru/nanoscan

\section{Autoren}

\section{Dipl.-Ing. (FH) Marcel Mück}

Technische Fachhochschule Wildau

Fachbereich Ingenieurwesen/Wirtschaftsingenieurwesen Labor für Oberflächentechnik

Telefon +493375 508-286

E-Mail: mmueck@igw.tfh-wildau.de

\section{Alexander Soshnikov}

Technological Institute for Superhard and Novel Carbon Materials

7a Centralnaja H., Troitsk, Moscow Reg.,142190 Russia

Telefon +70953340855

E-Mail: nanoscan@mtu-net.ru

\section{Dr. Bodo Wolf}

Technische Fachhochschule Wildau

Fachbereich Ingenieurwesen/Wirtschaftsingenieurwesen Labor für Oberflächentechnik

Telefon +493375 508-217

E-Mail: bwolf@igw.tfh-wildau.de

\section{Prof. Dr. Asta Richter}

Technische Fachhochschule Wildau

Fachbereich Ingenieurwesen/Wirtschaftsingenieurwesen Labor für Oberflächentechnik

Telefon +493375 508-219

E-Mail: richter@pt.tfh-wildau.de 